\title{
Effects of sildenafil and calcitonin gene- related peptide on brainstem glutamate levels: a pharmacological proton magnetic resonance spectroscopy study at 3.0 T
}

\author{
Samaira Younis ${ }^{1}$, Anders Hougaard ${ }^{1}$, Casper Emil Christensen'1 , Mark Bitsch Vestergaard², Esben Thade Petersen²,
} Olaf Bjarne Paulson ${ }^{4}$, Henrik Bo Wiberg Larsson ${ }^{2}$ and Messoud Ashina ${ }^{1 *}$

\begin{abstract}
Background: Studies involving human pharmacological migraine models have predominantly focused on the vasoactive effects of headache-inducing drugs, including sildenafil and calcitonin gene-related peptide (CGRP). However, the role of possible glutamate level changes in the brainstem and thalamus is of emerging interest in the field of migraine research bringing forth the need for a novel, validated method to study the biochemical effects in these areas.

Methods: We applied an optimized in vivo human pharmacological proton $\left({ }^{1} \mathrm{H}\right)$ magnetic resonance spectroscopy (MRS) protocol (PRESS, repetition time $3000 \mathrm{~ms}$, echo time 37.6-38.3 ms) at 3.0 T in combination with sildenafil and CGRP in a double-blind, placebo-controlled, randomized, double-dummy, three-way cross-over design. Seventeen healthy participants were scanned with the ${ }^{1} \mathrm{H}$-MRS protocol at baseline and twice (at 40 min and $140 \mathrm{~min}$ ) after drug administration to investigate the sildenafil- and CGRP-induced glutamate changes in both brainstem and thalamus.

Results: The glutamate levels increased transiently in the brainstem at 40-70 min after sildenafil administration compared to placebo $(5.6 \%, P=0.039)$. We found no sildenafil-induced glutamate changes in the thalamus, and no CGRP-induced glutamate changes in the brainstem or thalamus compared to placebo. Both sildenafil and CGRP induced headache in $53 \%-62 \%$ of participants. We found no interaction in the glutamate levels in the brainstem or thalamus between participants who developed sildenafil and/or CGRP-induced headache as compared to participants who did not.

Conclusions: The transient sildenafil-induced glutamate change in the brainstem possibly reflects increased excitability of the brainstem neurons. CGRP did not induce brainstem or thalamic glutamate changes, suggesting that it rather exerts its headache-inducing effects on the peripheral trigeminal pain pathways.
\end{abstract}

Keywords: MRS, Glutamate, Glx, Lactate, Migraine, Brainstem, Thalamus, CGRP, Sildenafil

\section{Background}

Human pharmacological migraine models have been used for the past two decades with great success to study migraine attack mechanisms using vasoactive drugs such as calcitonin gene-related peptide (CGRP) and sildenafil [1-7]. The models have been pivotal in the development of new anti-migraine therapy [8].

\footnotetext{
* Correspondence: ashina@dadlnet.dk

'Danish Headache Center, Department of Neurology, Rigshospitalet Glostrup, University of Copenhagen, Copenhagen, Denmark

Full list of author information is available at the end of the article
}

Human pathophysiological studies applying these models have predominantly focused on the cerebrovascular effects of the headache-inducing substances. However, emerging evidence suggests that metabolic changes, especially of brain glutamate levels $[9,10]$, in the brainstem [11-15] and thalamus [15] are key processes for the initiation of migraine headache attacks and thereby potentially important effects of the headache-inducing drugs. At present, methods for the study of pharmacologically induced biochemical effects on the brainstem glutamate levels have not been validated. 
Pharmacological proton $\left({ }^{1} \mathrm{H}\right)$ magnetic resonance spectroscopy (MRS) provides the ability to non-invasively study drug-induced biochemical changes in the brain. Imaging of the deep brain structures, especially the brainstem by magnetic resonance imaging (MRI), is challenging due to the small size of the region of interest, location in areas of relatively high magnetic field inhomogeneity and potential physiological artifacts. Thus, it is essential to systematically investigate the quality and reproducibility of ${ }^{1} \mathrm{H}$-MRS measurements in these areas before application of the method in patients. Only a few ${ }^{1} \mathrm{H}$-MRS studies of the brainstem have previously been conducted. One such study, of patients with amyotrophic lateral sclerosis, did not report data on the reproducibility or variability of the glutamate measurements [16], while other ${ }^{1} \mathrm{H}$-MRS brainstem studies did not measure the glutamate concentrations at all [17-20]

The headache-inducing drugs, CGRP and sildenafil, were selected for the study based on their different modes of action. CGRP is generally considered to exert its primary effect outside of the central nervous system (CNS), in the meningeal vasculature and the first order trigeminal neurons [21, 22], while sildenafil, as a lipophilic molecule, readily crosses the blood-brain barrier [23].

Here, we conducted a double-blind, placebo-controlled, randomized, double-dummy, three-way cross-over pharmacological ${ }^{1} \mathrm{H}$-MRS study to investigate the sildenafil- and CGRP-induced glutamate concentration changes in healthy participants. Our null-hypothesis was that the glutamate levels are not altered in the brainstem of healthy participants after administration of sildenafil and CGRP when compared to placebo. Additionally, we assessed the spectral quality and variability of the glutamate measurements over time in the brainstem based on our ${ }^{1} \mathrm{H}$-MRS protocol.

\section{Methods}

\section{Participants}

Healthy volunteers were recruited through announcement on a Danish website for recruitment of participants to health research (www.forsoegsperson.dk). Inclusion criteria were: age $18-50$ years and weight $50-$ $100 \mathrm{~kg}$. Exclusion criteria were: history of any primary headache disorders (except episodic tension-type headache for $<2$ day per month during the last year) according to the diagnostic criteria of the beta version of the third International Classification of Headache Disorders (ICHD-3 beta) [24], first-degree family members with migraine or other primary headache disorders according to ICHD-3 beta (except episodic tension-type headache for $<6$ days per month), daily intake of medication (except oral contraceptives), no usage of safe contraception, cardiovascular, cerebrovascular, or psychiatric disease, and drug abuse. Participants were excluded if there were any contraindications to MRI such as metal implants, pacemaker, insulin pump, claustrophobia and/or surgical procedure during the last 6 weeks before inclusion. We also excluded participants with braces and teeth implants of metal, which are normally regarded MRI compatible, to avoid potential MR scan artifacts in the deep brain structures of interest.

\section{Experimental design}

All participants were randomly allocated to receive sildenafil, CGRP and placebo on three separate study days. On each study day, participants underwent an MRI scan protocol consisting of three scan sessions: a baseline MRI scan, followed by two additional post drug administration MRI scan sessions. The first post drug scan was initiated at $40 \mathrm{~min}$ (scan 1), and the second scan was initiated at $140 \mathrm{~min}$ (scan 2) after administration of sildenafil, CGRP or placebo (Fig. 1). MR spectra were obtained from brainstem and thalamus during each scan.

On the sildenafil day, the participants received sildenafil as two $50 \mathrm{mg}$ tablets (STADA, Bad Vilbel, Germany) in two non-transparent capsules, combined with placebo isotonic saline infusion into the cubital vein for $20 \mathrm{~min}$ (Pressure tubes, Argon Medical Devices, The Hague, the Netherlands), at the time of infusion start. On the CGRP day, the participants received $1.5 \mu \mathrm{g} / \mathrm{min}$ human-alfa-CGRP (PolyPeptide, Strasbourg, France) via infusion for $20 \mathrm{~min}$ combined with placebo calcium in two non-transparent capsules. On the placebo day, the participants received placebo isotonic saline infusion for $20 \mathrm{~min}$ combined with placebo calcium in two non-transparent capsules. The sildenafil and CGRP dosages for the study were

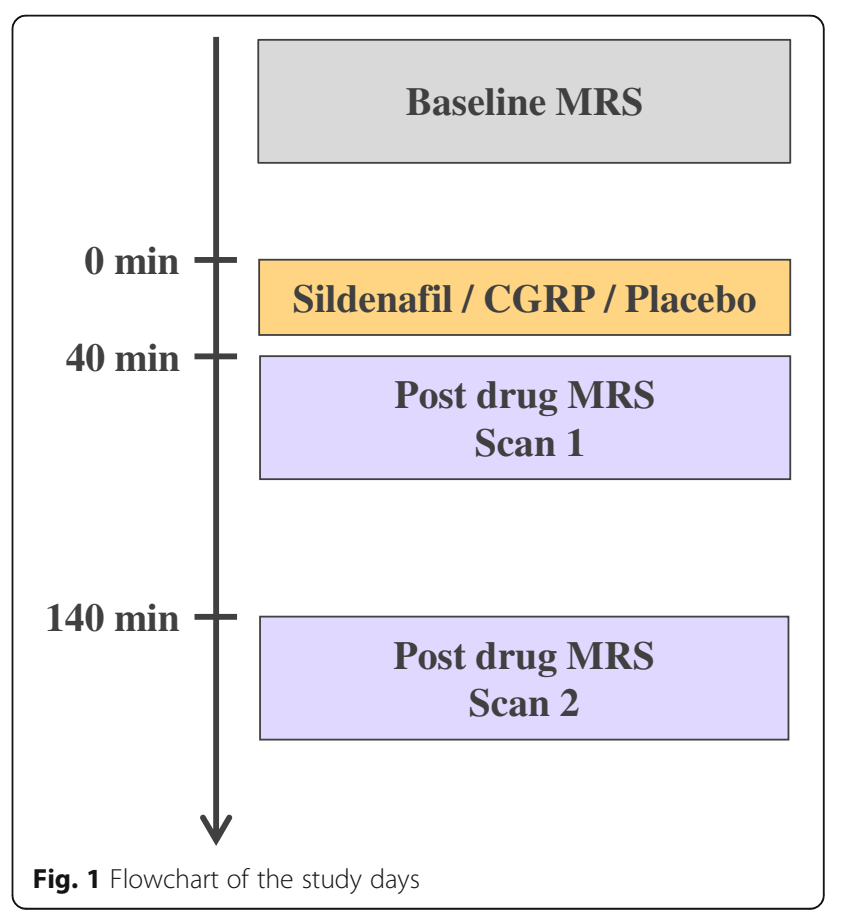


determined based on findings of previous studies, which reported sildenafil- and CGRP-induced headache in healthy volunteers, and migraine-like attack in migraine patients [1-7]. The randomization was administrated by the Hospital Pharmacy of the Capital Region of Denmark.

All participants were headache-free for at least $72 \mathrm{~h}$ before each study day. The participants were not allowed coffee, tea, cocoa, soft drinks, alcohol or tobacco for $12 \mathrm{~h}$ before study start on each study day, and fasted for all food and beverages (except for water), for $4 \mathrm{~h}$ before study start. Between scan 1 and scan 2, all participants were offered a standardized small meal consisting of soft bread with cheese, banana, and water. Other criteria for the study days were no intake of any medication four half-lives before the start of the study day, except for oral contraception. After insertion of a peripheral venous catheter (18G Vasofix ${ }^{\circ}$ Safety, B.Braun, Melsungen, Germany) into a cubital vein, the participants were instructed to rest in a hospital bed for approximately $30 \mathrm{~min}$ before the baseline scans.

We aimed to initiate the scan sessions at the same time of the day on all three study days for each participant, allowing for a maximum time deviation of $1 \mathrm{~h}$, to account for metabolite concentration variations due to the circadian rhythm $[25,26]$. In addition, the timing of scan 1 and scan 2 was fixed according to the baseline scan. The ${ }^{1} \mathrm{H}$-MRS sequences were part of a larger study (results of these will be presented elsewhere). Before and after each scan sequence it was ensured that participants remained awake, and data were excluded in case they fell asleep during the scans as this could affect the measurements [27]. Participants were instructed to remain still and avoid any head motion during the scan sessions to ensure stable measurements from the regions of interest.

\section{Headache characteristics}

Data on headache characteristics were acquired on each study day, i.e. intensity, quality, aggravation by physical activity, location and associated symptoms (nausea, photophobia, and phonophobia). The headache intensity was rated on a numeric rating scale ranging from 0 to 10 , where ' 0 ' translated to no headache and ' 10 ' to the worst imaginable headache. The headache data were obtained between all scans. All participants were asked to register headache hourly in a standardized questionnaire after the last scan session until $24 \mathrm{~h}$, starting from the time of study drug administration.

\section{Vital signs}

The vital variables were registered and monitored at baseline, and during the scan sessions after study drug administration. Systolic and diastolic blood pressure were measured with an interval of $10 \mathrm{~min}$, and heart rate, blood oxygen saturation and nostril end-tidal $\mathrm{CO}_{2}$ tension (water trap and gas sample line, Medrad, Warrendale, PA) (Veris Monitor, Medrad, Warrendale, PA) were monitored continuously.

\section{Data acquisition and imaging protocol}

All MRI scans were performed on a 3.0 T Philips Achieva MRI scanner (Philips Medical Systems, Best, The Netherlands) using a 32-channel phase array head coil.

\section{Anatomical scan}

High-resolution anatomical scans were obtained with a 3D T1-weighted turbo field echo sequence (field of view $240 \times 240 \times 170 \mathrm{~mm}^{3}$; voxel size $1.00 \times 1.08 \times 1.10 \mathrm{~mm}^{3}$; echo time $3.7 \mathrm{~ms}$; repetition time $8.0 \mathrm{~ms}$; flip angle $8^{\circ}$ ). The reconstruction software on the scanner was used to additionally obtain the axial and coronal anatomical views of the scan to ensure correct placement of the volumes-of-interest (VOIs) for brainstem and thalamus.

\section{Magnetic resonance spectroscopy}

We used proton $\left({ }^{1} \mathrm{H}\right)$ magnetic resonance spectroscopy (MRS) to measure the combined concentration of glutamate and glutamine (reported as 'glutamate'), lactate, $\mathrm{N}$-Acetylaspartate (NAA) and the total concentration of creatine i.e. phosphocreatine and creatine. The water-suppressed point-resolved spectroscopy (PRESS) pulse sequence was used in brainstem (repetition time $3000 \mathrm{~ms}$; echo time $38.3 \mathrm{~ms}$, voxel size $10.5 \times 12.5 \times$ $22 \mathrm{~mm}^{3} ; 480$ acquisitions; total duration $24 \mathrm{~min}$ ) and thalamus (repetition time $3000 \mathrm{~ms}$; echo time $37.6 \mathrm{~ms}$; voxel size $16 \mathrm{~mm} \times 12 \mathrm{~mm} \times 12 \mathrm{~mm} ; 192$ acquisitions; total duration 9 min $36 \mathrm{~s}$ ). High number of acquisitions was used to ensure sufficient signal-noise-ratio. Voxel size based shimming was performed using first-order pencil beam to reduce the inhomogeneity in the chosen VOIs. The protocol was thus optimized to precisely target small VOIs in deep brain structures and to avoid cerebrospinal fluid contributions and partial volume artifacts. The repetition time was $3000 \mathrm{~ms}$ to ensure sufficient relaxation. The unsuppressed water signal was measured from the VOIs and used as internal reference for quantification [28]. The first VOI was placed unilaterally in the right side of the brainstem, and the second VOI was placed in the left, contralateral thalamus, following the anatomical and functional trigeminal pain pathways.

\section{Metabolite quantification and analysis}

Post-processing and quantification of the spectral data were performed by LCModel (Version 6.3-1F, Toronto, Canada). Representative ${ }^{1} \mathrm{H}$-MRS spectra obtained from brainstem and thalamus are illustrated in Fig. 2. Spectra were evaluated in a blinded manner and abnormal 

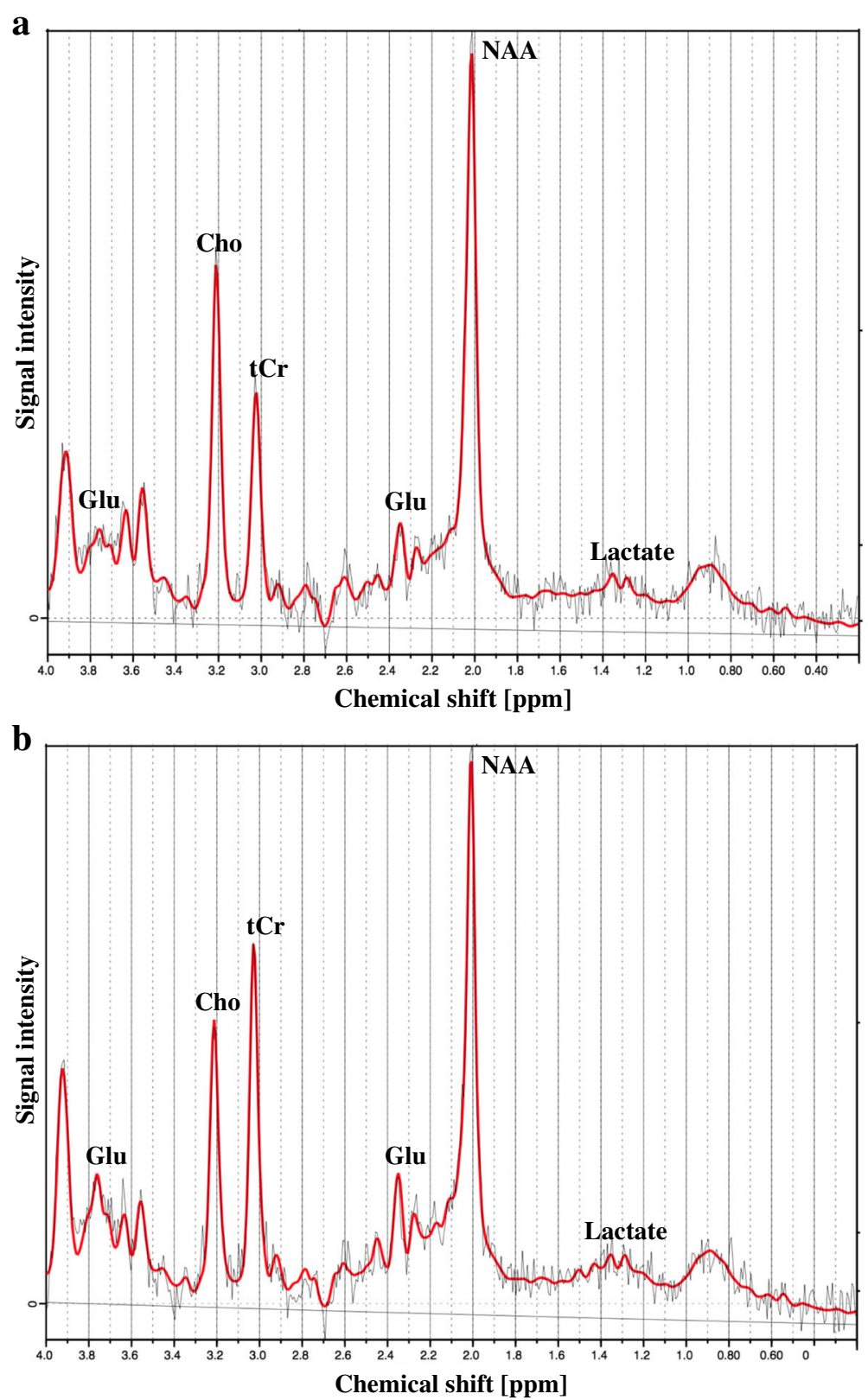

Fig. 2 MR spectra from brainstem and thalamus. Examples of (a) brainstem and (b) thalamus spectra are obtained at baseline with the point-resolved spectroscopy (PRESS) pulse sequence at $3.0 \mathrm{~T}$. The spectra are acquired from LCModel. The red line represents the fit, and the horizontal linear line represents the baseline as estimated by LCModel. Cho: Choline, Glu: Glutamate, tCr: Total creatine, NAA: N-Acetylaspartate

spectra were excluded. The quality of the included spectra was estimated based on the signal-noise-ratio (SNR) and full-width of half-maximum (FWHM) of the spectra peaks as provided by LCModel. The means and standard deviations of the SNR and FWHM for the brainstem and thalamus spectra were calculated.

\section{Statistical analysis}

The primary endpoint was glutamate, lactate, NAA and total creatine concentration changes in brainstem and thalamus from baseline to after sildenafil and CGRP administration, compared to the corresponding placebo changes. A linear mixed model was used for each metabolite with interaction between scans (baseline, scan 1 and scan 2) and drug days (sildenafil, CGRP and placebo) and with subjects and study day (5 levels) nested within subjects as random effects. The placebo day baseline scan was set as the reference parameter in the model.

The secondary endpoint was changes in the metabolite concentrations in participants who developed 
pharmacologically induced headache during the scan sessions after sildenafil and CGRP, compared to participants who did not. A linear mixed model was used for each metabolite on the sildenafil and CGRP day with interaction between scans and headache and the random effects: subjects and study day nested within subjects. The data did not allow for correlation analyses between metabolite concentration and headache characteristics. The headache frequencies after sildenafil and CGRP were compared to placebo using McNemar's test.

For explorative vital parameter analyses, we included data from the following time points: $0,20,70,120$, and 170 min after infusion. Changes from baseline after sildenafil and CGRP were compared to placebo using a linear mixed model with interaction between drug days and the selected time points with subjects as random effects.

The variability structure of the glutamate measurements in the brainstem and thalamus was estimated in an explorative analysis based on baseline, scan 1, and scan 2 data acquired on the placebo day, and baseline data acquired on the sildenafil and CGRP day, using a linear mixed model with no fixed effects, and the random effects: subjects and study day (5 levels) nested within subjects.

All statistical analyses were performed using $\mathrm{R}$ (Version 3.4.2). $P$ values were reported as two-tailed with a level of significance of $5 \%$.

\section{Results}

\section{Participants}

Seventeen healthy volunteers participated in the study (10 women and 7 men) with mean age 22.9 ( $\mathrm{SD} \pm 3.4$ and range 18-30 years) (Fig. 3). Vitals signs are presented in Fig. 4.

\section{Alterations in metabolite concentration Brainstem}

The glutamate concentration significantly increased from baseline to scan 1 after sildenafil compared to the corresponding change after placebo $(P=0.039)$ (Table 1 , Fig. 5). The lactate concentration decreased from baseline to scan $1(P=0.017)$, but not to scan $2(P=0.156)$ after sildenafil, compared to corresponding changes after placebo. In the brainstem, we did not detect changes in the metabolite concentrations from baseline to scan 1 or scan 2 after CGRP, compared to placebo.

\section{Thalamus}

We did not detect changes in the glutamate, lactate or NAA concentrations in the thalamus from baseline to scan 1 or scan 2 after sildenafil or CGRP, compared to placebo. The increase in the total creatine concentration from baseline to scan 2 after CGRP $(3.3 \%, P=0.028)$ was significant in comparison to placebo $(P=0.004)$.

\section{Headache vs. no headache}

The proportion of participants who developed headache during scan 1 and scan 2, and after the scan sessions and until $24 \mathrm{~h}$ from drug administration, is reported in Fig. 6. We found no interaction in the glutamate, lactate, NAA or total creatine concentrations in the brainstem or thalamus between participants who developed sildenafil- and/or CGRP-induced headaches as compared to participants who did not.

\section{Quality of spectra}

The brainstem spectra had mean SNR of $17.56( \pm 2.33)$, and mean FWHM of $0.05 \mathrm{ppm}( \pm 0.01) / 6.39 \mathrm{~Hz}( \pm 1.28)$. In thalamus, the mean SNR was $15.30( \pm 1.86)$ and the mean FWHM was $0.04 \mathrm{ppm}( \pm 0.01) / 5.11 \mathrm{~Hz}( \pm 1.28)$. In addition, the Cramér-Rao lower bound was $<12 \%$ for glutamate measurements in the brainstem and thalamus, except for $12-13 \%$ in four brainstem spectra and one thalamus spectrum in different subjects.

\section{Glutamate variability in brainstem and thalamus}

From the linear mixed model, we obtained separate brainstem glutamate concentration variations, where $6.9 \%$ was due to residual measurement error with additional $2.1 \%$ due to inter-subject variation, and $6.0 \%$ due to between day variations. The thalamic glutamate concentration variations were $6.8 \%$ due to residual measurement error with $2.7 \%$ inter-subject and $0 \%$ between day variations.

The mean time difference from day 1 to day 2 of the study days was 12.5 days $( \pm 9.2)$ and 10.7 days $( \pm 6.0)$ between day 2 and day 3. Participants were mainly scanned from afternoon time on all three scan days. The scans were initiated in the morning for three subjects, whereof one subject completed all three study days.

\section{Discussion}

The major outcome of the present study was an increase in the glutamate concentration in the brainstem after administration of sildenafil when compared to placebo. We did not detect any changes in the glutamate concentration in the brainstem after CGRP infusion.

\section{Sildenafil-induced biochemical changes}

Glutamate, as the major excitatory neurotransmitter in the brain, promotes neuronal depolarization [29]. Extracellular glutamate levels are directly correlated to levels of neuronal hyperexcitability and seizure intensity in animal models of epilepsy [30,31]. Here, we evaluated the combined concentration of the glutamate and glutamine as these metabolites are not differentiable at $3.0 \mathrm{~T}{ }^{1} \mathrm{H}$-MRS. In healthy volunteers, the majority of the combined concentration consists of glutamate $(\sim 80 \%)$ [32] and $13 \%-22 \%$ of the glutamate concentration in the healthy brain is present in the 


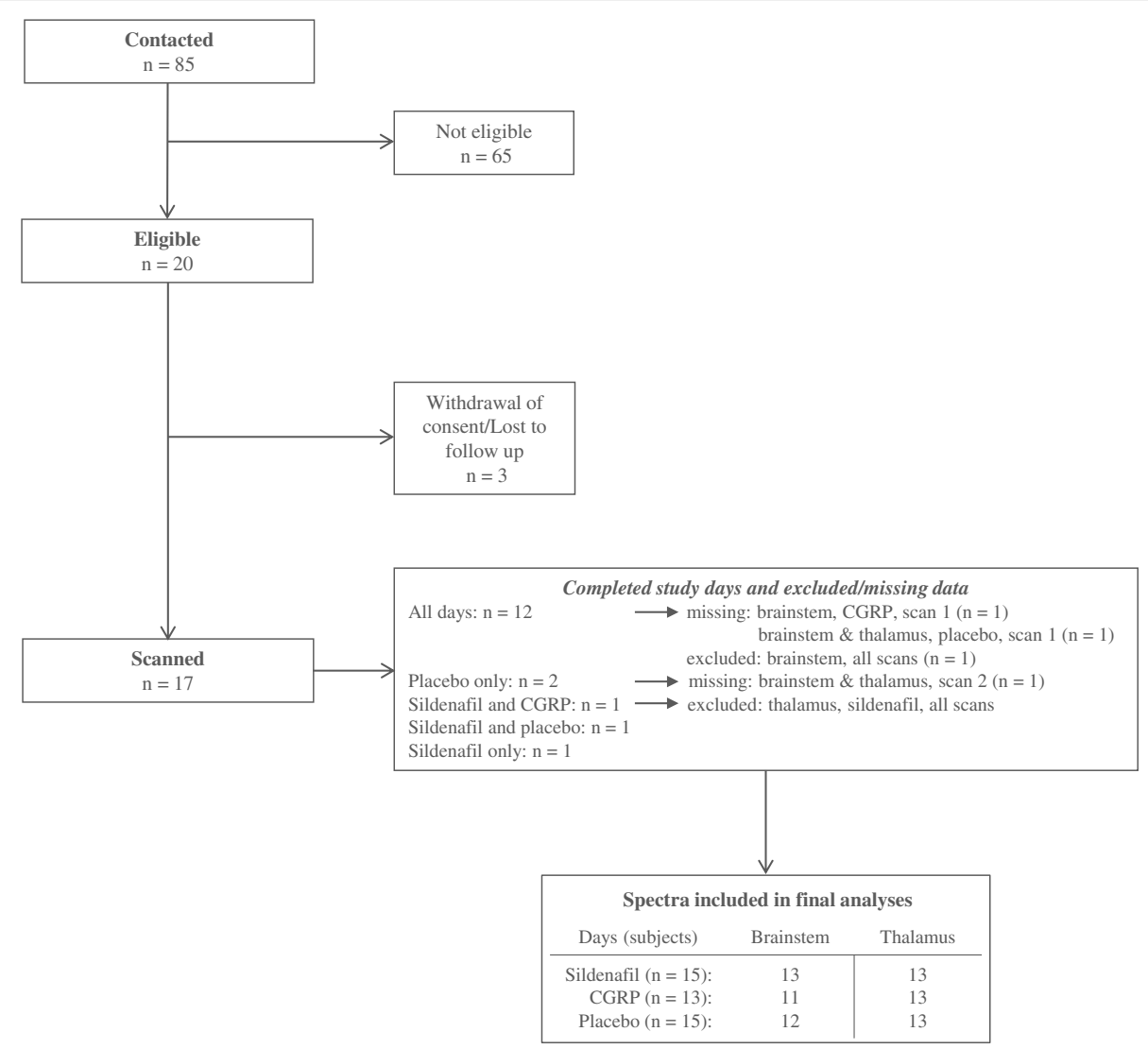

Fig. 3 Flowchart of the inclusion process of participants and data for analyses. Seventeen participants were scanned, whereof 12 completed all three study days. The remaining participants completed 1-2 study days. One subject completed only the placebo day due to loss to follow up. One subject withdrew after scan 1 on the first study day (placebo) due to claustrophobia, thus scan 2 data are missing. One subject completed the sildenafil and CGRP day, but thalamus data were excluded from the sildenafil day as the subject fell asleep during the thalamus baseline scan. One subject did not complete the CGRP day due to loss to follow up. One subject only participated on the sildenafil day due to finalization of the study. Data were further missing due to technical issues: scan 1 data (brainstem and thalamus) after placebo from one subject, and scan 1 brainstem data after CGRP from another subject. Brainstem data were excluded from one subject for all three study days due to poor spectral quality. n: Number

extracellular space [29]. Most likely, the glutamate concentrations measured by ${ }^{1} \mathrm{H}$-MRS largely reflect the extracellular glutamate levels. In support of this, a ${ }^{1} \mathrm{H}$-MRS study reported lower glutamate levels in amyotrophic lateral sclerosis patients treated with riluzole, a drug that increases glutamate uptake in central nervous system (CNS) neurons, compared to riluzole-naive amyotrophic lateral sclerosis patients and healthy controls [16]. The transient sildenafil-induced increase of glutamate in the brainstem in the present study thus likely reflects increased extracellular glutamate levels and possibly increased neuronal excitability. In support, sildenafil is able to cross the blood-brain barrier $[23,33]$ and some individuals report CNS side effects, such as dizziness and confusion [33-36]. Thus, sildenafil may be able to directly affect the neurons in deep brain structures such as the brainstem. In contrast, a functional MRI (fMRI) study of the visual cortex suggested that oral sildenafil intake did not change the neuronal activation threshold either at 1 or $2 \mathrm{~h}$ after administration [2]. The plasma $t_{\max }$ of oral $100 \mathrm{mg}$ sildenafil is about $1 \mathrm{~h}$ with a close to $4 \mathrm{~h}$ half-life in the fasting state [36]. Here, we detected an increased glutamate level at scan 1 (40-70 min after sildenafil), around the time of $t_{\max }$, but not at scan 2 (140-170 min after sildenafil). Possibly, plasma concentrations of sildenafil above a certain level are needed to alter the glutamate levels. Another possibility is that the transient changes may be attributed to adaptation of sildenafil's effect at scan 2.

We detected no difference in the glutamate levels between groups of participants developing headache vs. no headache. It should be noted that the participants were healthy with no family history of migraine developing merely a mild to moderate non-migraine headache after the drug administration. Therefore, we speculate that a "healthy" trigeminonociceptive system would not be sufficiently activated to produce detectable changes in the glutamate level. This may also explain the lack of changes in the glutamate levels after CGRP as well as in the thalamus. 


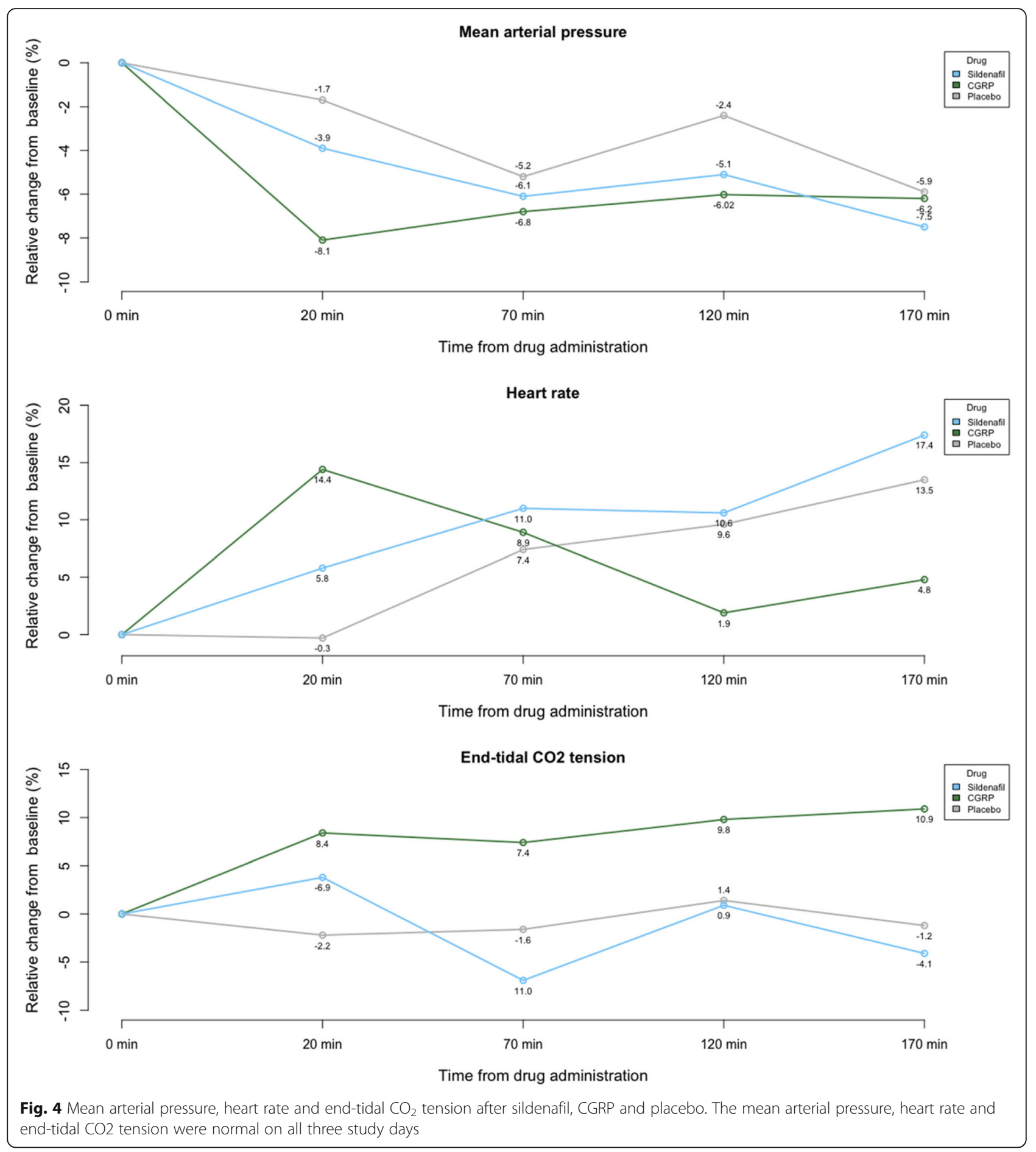

Given the transient glutamate changes and lack of correlation to headache status, it is likely that the observed changes are related to the pharmacological effects of the drug rather than the headache per se.

The lactate concentration was decreased in the brainstem at scan 1 after sildenafil compared to the corresponding placebo change. This observation is very interesting since brain lactate levels under normal conditions increase during neuronal activation [37]. Therefore, we would expect the brainstem lactate levels to increase following sildenafil administration, along with the observed increase in glutamate. A possible explanation could be that the lactate decrease reflects a neuronal energy consumption via conversion to pyruvate [38]. The lactate concentration finding in the present study should be interpreted with caution due to the relatively large standard deviations. Also of note, the 
Table 1 Summary of metabolite concentrations in brainstem after sildenafil, CGRP and placebo

\begin{tabular}{|c|c|c|c|c|c|c|c|c|c|c|}
\hline & \multicolumn{2}{|l|}{ Baseline } & \multicolumn{4}{|l|}{ Scan 1} & \multicolumn{4}{|l|}{ Scan 2} \\
\hline & Mean mmol/L & SD & Mean mmol/L & SD & $\%$ change from baseline & $P$ & Mean mmol/L & SD & $\%$ change from baseline & $P$ \\
\hline \multicolumn{11}{|l|}{ Glutamate } \\
\hline Sildenafil & 7.77 & 0.65 & 8.21 & 0.53 & 5.6 & $0.039^{*}$ & 8.06 & 0.77 & 3.7 & 0.101 \\
\hline CGRP & 7.92 & 1.11 & 7.49 & 0.88 & -5.4 & 0.639 & 8.08 & 0.73 & -2.0 & 0.228 \\
\hline Placebo & 7.96 & 0.50 & 7.72 & 0.61 & -3.0 & - & 7.70 & 0.65 & -3.3 & - \\
\hline \multicolumn{11}{|l|}{ Lactate } \\
\hline Sildenafil & 0.90 & 0.21 & 0.45 & 0.41 & -50.0 & $0.017^{*}$ & 0.43 & 0.33 & -51.9 & 0.156 \\
\hline CGRP & 0.71 & 0.51 & 0.49 & 0.44 & -30.9 & 0.151 & 0.42 & 0.41 & -40.6 & 0.494 \\
\hline Placebo & 0.75 & 0.65 & 0.89 & 0.68 & 21.6 & - & 0.62 & 0.42 & -15.6 & - \\
\hline \multicolumn{11}{|l|}{ NAA } \\
\hline Sildenafil & 7.73 & 0.79 & 7.93 & 0.79 & 2.8 & 0.236 & 7.95 & 0.69 & 3.0 & 0.370 \\
\hline CGRP & 7.58 & 0.72 & 7.56 & 0.68 & -0.2 & 0.821 & 8.06 & 0.82 & 6.3 & 0.127 \\
\hline Placebo & 7.61 & 0.63 & 7.64 & 0.58 & 0.4 & - & 7.73 & 0.72 & 1.6 & - \\
\hline \multicolumn{11}{|c|}{ Total creatine } \\
\hline Sildenafil & 4.25 & 0.35 & 4.33 & 0.32 & 1.0 & 0.978 & 4.23 & 0.28 & -0.3 & 0.735 \\
\hline CGRP & 4.38 & 0.38 & 4.39 & 0.53 & 0.03 & 0.562 & 4.50 & 0.33 & 2.7 & 0.170 \\
\hline Placebo & 4.24 & 0.33 & 4.33 & 0.47 & 2.0 & - & 4.21 & 0.32 & -0.7 & - \\
\hline
\end{tabular}

${ }^{*} P<0.05$. $P$ values reported for delta change from baseline to scan 1 and 2 after sildenafil and CGRP, compared to the corresponding change from baseline after placebo NAA N-Acetylaspartate, SD standard deviation

lactate concentration is very low in the healthy brain (below $1.0 \mathrm{mmol} / \mathrm{L}$ ) [39]. This contributes to the risk of lactate signal loss in the spectrum due to chemical shift displacement or J-modulations deviations during the MRS measurements, which are known issues [39].

\section{CGRP-induced biochemical changes}

We detected no alterations in the glutamate levels after CGRP infusion in either brainstem or thalamus in healthy participants. This suggests that CGRP does not modify the neuronal excitability in these key CNS structures involved in pain processing in healthy subjects. The blood-brain barrier is believed to have no permeability to CGRP $[4,40]$, and thus little or no direct effects on central brain regions, which our findings support.

In line with previous reports [3, 4], we found that participants developed more headache after CGRP, compared to placebo, demonstrating that CGRP is able to activate the trigeminal pain pathway. Given that systemic CGRP is unlikely to cross the blood-brain barrier, the present findings support the notion that CGRP acts on perivascular afferents [22] or the trigeminal ganglion $[21,41]$. Interestingly, an fMRI study reported no change in the neuronal activation of the visual cortex of healthy volunteers after CGRP infusion [42]. Increased neuronal response to visual stimulation has previously been

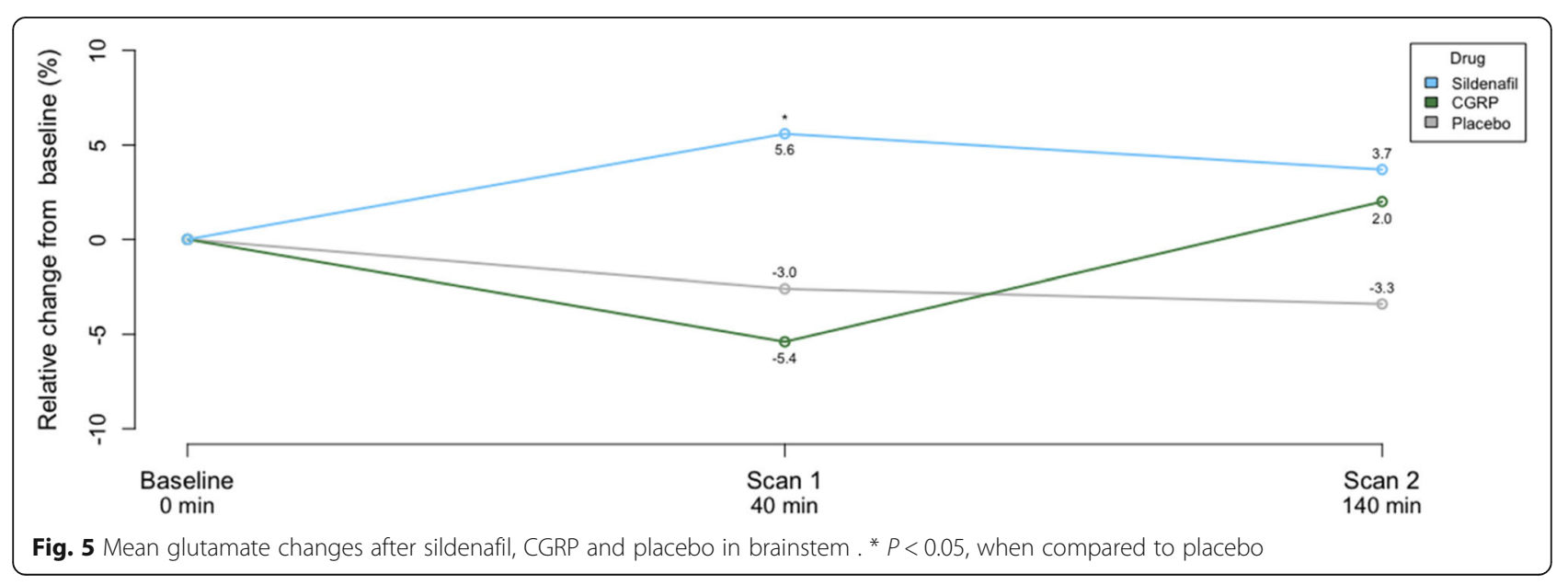




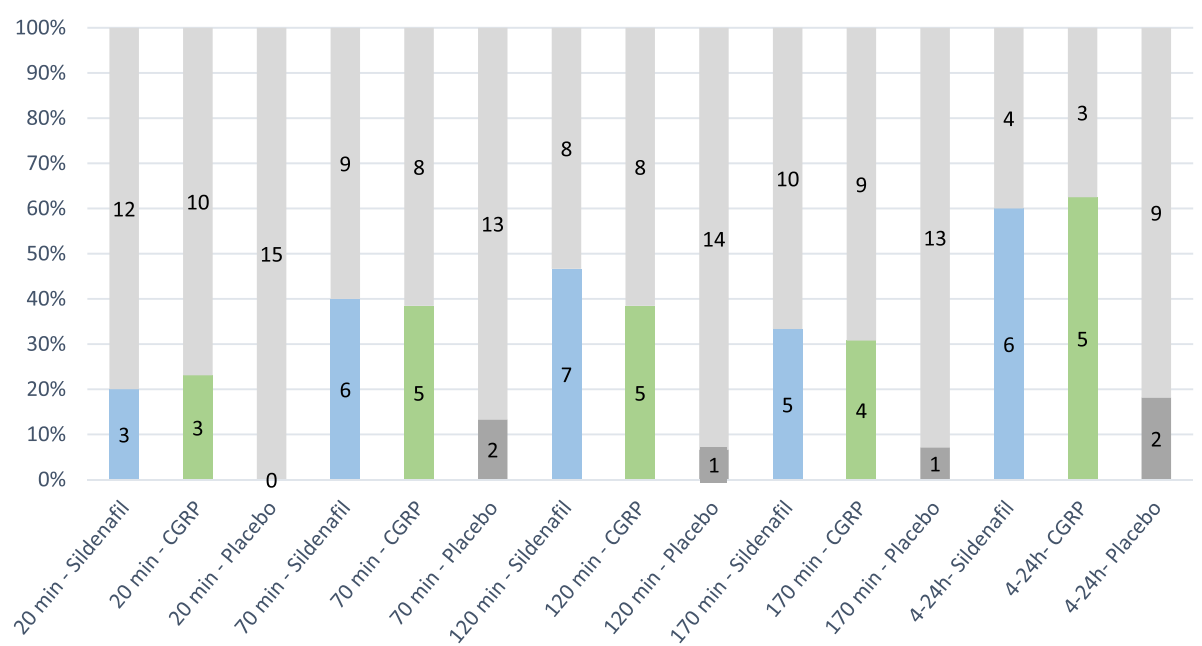

Fig. 6 Proportion of healthy participants who developed headache after sildenafil, CGRP and placebo. Blue, green and dark grey bars indicate headache. Light grey bars indicate no headache. During the scan sessions (0-4 h), 8 of 13 participants (62\%) developed headache after CGRP ( $P$ $=0.041$, compared to placebo), 8 of $15(53 \%)$ developed headache after sildenafil ( $P=0.131$, compared to placebo), and 2 of 13 (13\%) developed headache after placebo.

shown to be correlated with an increase in the glutamate levels $[43,44]$. Another fMRI study involving application of heat pain to the forehead of healthy volunteers reported altered blood-oxygenation-level-dependent signal 40 min after administration of CGRP in pain associated brain regions, including the brainstem and thalamus, with no changes during placebo [45]. This observation suggests that CGRP may be capable of modulating the neuronal response indirectly (i.e. outside the CNS) given that the pain pathway is already activated [45].

\section{Reliability of glutamate measurements}

With our ${ }^{1} \mathrm{H}$-MRS protocol, we obtained high quality spectra from the brainstem and thalamus with narrow line widths and relatively high SNR allowing for reliable quantification. A previous $3.0 \mathrm{~T}^{1} \mathrm{H}$-MRS study measured glutamate changes in the brainstem without reporting the SNR or spectral line widths, but visual inspection of the brainstem spectrum reveals more noise compared to the brainstem spectra obtained in the present study [16]. Other ${ }^{1} \mathrm{H}$-MRS brainstem studies reported relatively wider mean line widths of $8.1 \mathrm{~Hz}( \pm 0.9)$ [18], and $10.04 \mathrm{~Hz}( \pm$ 4.64) [17] at $3.0 \mathrm{~T}$, and $7 \mathrm{~Hz}$ at $4.0 \mathrm{~T}$ [19] indicating spectra of lower quality. One of the studies reported the SNR as well, which was relatively high, $21( \pm 3)$, most likely due to the larger VOI used in the study [18]. None of the studies reported glutamate findings and the VOIs were larger than in the present study [17-19]. While large VOIs can improve the spectral quality, it also restricts the possibility of targeting a brain area with precision.

A previous $3.0 \mathrm{~T}^{1} \mathrm{H}$-MRS study of thalamus used a larger VOI with fewer acquisitions, and reported a mean line width similar to the present study findings, but did not report the SNR value for comparison [17]. One $1.5 \mathrm{~T}{ }^{1} \mathrm{H}$-MRS thalamus study reported reduced mean line width of $3.2 \mathrm{~Hz}( \pm 0.5)$, however, the SNR of $3.9( \pm$ 1.2) was much lower [46].

The brainstem ${ }^{1} \mathrm{H}$-MRS spectra reveal a different metabolite composition compared to the conventional spectra obtained from e.g. the thalamus and occipital lope, as the choline peak is higher than the total creatine peak (Fig. 2), which is commonly reported [16, 17, 19].

To our knowledge, our study is the first ${ }^{1} \mathrm{H}$-MRS study to provide information on the variability of glutamate levels in both brainstem and thalamus, based on repeated measurements, on the same day and on three separate days. In the present study, the overall variability of the glutamate measurements was low. For comparison, a previous $3.0 \mathrm{~T}{ }^{1} \mathrm{H}$-MRS study reported a higher inter-subject glutamate variability of $15.4 \%-16.3 \%$ in the deep brain area of the amygdala, based on two scans obtained 1 week apart [47]. Another $3.0 \mathrm{~T}^{1} \mathrm{H}$-MRS study of repeated measurements on three consecutive days reported residual measurement error as the main contributor to the glutamate variability in a small hippocampus VOI [48]. Finally, one previous $7.0 \mathrm{~T}$ ${ }^{1} \mathrm{H}$-MRS study reported higher glutamate variability of $11.48 \%( \pm 8.87)$ within day (based on two scans), and $6.56 \%( \pm 4.69)$ between day, measured in the visual cortical area of healthy subjects [49]. However, the study did not report a separate inter-subject and residual measurement error variability [49].

The present study has several major strengths to account for the measurement error variation, as all 
participants were scanned at fixed time points on each scan day, accounting for possible changes due to the metabolic circadian rhythm $[25,26]$. In addition, we maintained identical and stable study conditions for all participants on all three study days, including detailed dietary restrictions before and during the scan sessions. All participants were carefully instructed to avoid any head motion during the scans. However, we cannot exclude the possibility of motion affecting our findings during the scan sessions. We estimated that the high acquisition number for the ${ }^{1} \mathrm{H}$-MRS sequences was appropriate and feasible to obtain a sufficient signal noise ratio from the spectral VOIs. Additionally, as our primary aim was to investigate and compare relative changes from baseline within subjects, these issues were unlikely to affect our results.

\section{Conclusion}

Here we present a protocol for pharmacological ${ }^{1} \mathrm{H}$-MRS at $3.0 \mathrm{~T}$ in the brainstem and thalamus, with good spectral quality, and overall low measurement variability. We demonstrated that sildenafil induces transiently increased glutamate levels in the brainstem, which suggest transiently increased excitability of the brainstem neurons. CGRP does not induce glutamate changes in the brainstem or thalamic neurons, suggesting that its headache-inducing effects are not mediated by biochemical changes in deep brain structures, but rather its effects on the peripheral trigeminal pain pathways.

\section{Abbreviations \\ ${ }^{1} \mathrm{H}$ : proton; CGRP: calcitonin gene-related peptide; CNS: central nervous system; fMRl: functional magnetic resonance imaging; FWHM: full-width of half- maximum; ICHD-3 beta: The diagnostic criteria of the beta version of the third International Classification of Headache Disorders; MRI: magnetic resonance imaging; MRS: magnetic resonance spectroscopy; NAA: N-Acetylaspartate; PRESS: point-resolved spectroscopy pulse sequence; SNR: signal-noise-ratio; VOI: volume-of-interest}

\section{Funding}

We thank the Lundbeck Foundation [grant number R155-2014-171] and the Research Foundation of Rigshospitalet [grant number E-23327-02]. Funding sources played no role in study design, data collection, analysis, interpretation, manuscript preparation, or submission.

\section{Availability of data and materials}

The data set supporting the conclusions is available upon request to the first/corresponding author.

\footnotetext{
Authors' contributions

SY drafted and revised the paper and contributed to study design, protocol development, participant enrolment, acquisition and processing of data, statistical analysis and interpretation of data. $\mathrm{AH}$ contributed to study design, protocol development, interpretation of data and revising manuscript for content. CEC contributed to protocol development, participant enrolment, acquisition of data, and revising manuscript for content. MBV contributed to study design, processing of data, statistical analysis, and revising manuscript for content. ETP and OBP contributed to study design and revising manuscript for content. HBWL contributed to study design, protocol development, statistical analysis, and revising manuscript for content. MA initiated the study and contributed to study design, protocol development,
}

data interpretation, and revision of the manuscript. All authors read and approved the final manuscript.

\section{Ethics approval and consent to participate}

The study was approved by the Danish National Committee on Health Research Ethics (H-15019063) and conducted according to the Declaration of Helsinki and to the regulations of the Danish Data Protection Agency. The study is registered at ClincalTrials.gov (NCT03143465). Written informed consent was obtained from all participants after detailed oral and written study information.

\section{Consent for publication}

Not applicable.

\section{Competing interests}

MA reports personal fees from Alder BioPharmaceuticals, Allergan, Amgen, Alder, Eli Lilly, Novartis and Teva. MA participated in clinical trials as the principal investigator for Alder ALD403-CLIN-011 (Phase 3b), Amgen 20,120,178 (Phase 2), 20,120,295 (Phase 2), 20,130,255 (OLE), 20,120,297 (Phase 3), GM-11 gamma-Core-R trials, Novartis CAMG334a2301 (Phase 3b), Amgen PAC1 20,150,308 (Phase 2a), Teva TV48125-CNS-30068 (Phase 3). MA has no ownership interest and does not own stocks of any pharmaceutical company. MA serves as associated editor of Cephalalgia, co-editor of the Journal of Headache and Pain. MA is President-elect of the International Headache Society and General Secretary of the European Headache Federation. The remaining authors report no conflicts of interest.

\section{Publisher's Note}

Springer Nature remains neutral with regard to jurisdictional claims in published maps and institutional affiliations.

\section{Author details}

${ }^{1}$ Danish Headache Center, Department of Neurology, Rigshospitalet Glostrup, University of Copenhagen, Copenhagen, Denmark. 'Functional Imaging Unit, Department of Clinical Physiology, Nuclear Medicine and PET, Rigshospitalet Glostrup, University of Copenhagen, Copenhagen, Denmark. ${ }^{3}$ Danish Research Centre for Magnetic Resonance, Centre for Functional and Diagnostic Imaging and research, Copenhagen University Hospital Hvidovre, Copenhagen, Denmark. ${ }^{4}$ Neurobiology Research Unit, Department of Neurology, Rigshospitalet, University of Copenhagen, Copenhagen, Denmark.

Received: 12 April 2018 Accepted: 7 June 2018

Published online: 18 June 2018

\section{References}

1. Kruuse C, Thomsen LL, Jacobsen TB, Olesen J (2002) The phosphodiesterase 5 inhibitor sildenafil has no effect on cerebral blood flow or blood velocity, but nevertheless induces headache in healthy subjects. J Cereb Blood Flow Metab 22:1124-1131

2. Kruuse C, Hansen AE, Larsson HBW, Lauritzen M, Rostrup E (2009) Cerebral haemodynamic response or excitability is not affected by sildenafil. J Cereb Blood Flow Metab 29:830-839

3. Asghar MS, Hansen AE, Kapijimpanga T, van der Geest RJ, van der Koning P, Larsson HBW et al (2010) Dilation by CGRP of middle meningeal artery and reversal by sumatriptan in normal volunteers. Neurology 75:1520-1526

4. Petersen KA, Lassen LH, Birk S, Lesko L, Olesen J (2005) BIBN4096BS antagonizes human alpha-calcitonin gene related peptide-induced headache and extracerebral artery dilatation. Clin Pharmacol Ther 77:202-213

5. Kruuse C, Thomsen LL, Birk S, Olesen J (2003) Migraine can be induced by sildenafil without changes in middle cerebral artery diameter. Brain 126:241-247

6. Lassen LH, Haderslev PA, Jacobsen VB, Iversen HK, Sperling B, Olesen J (2002) CGRP may play a causative role in migraine. Cephalalgia 22:54-61

7. Asghar MS, Hansen AE, Amin FM, van der Geest RJ, Van Der KP, Larsson HBW et al (2011) Evidence for a vascular factor in migraine. Ann Neurol 69:635-645

8. Ashina M, Hansen JM, á Dunga BO, Olesen J (2017) Human models of migraine - short-term pain for long-term gain. Nat Rev Neurol 13:713-724

9. Hoffmann J, Charles A (2018) Glutamate and its receptors as therapeutic targets for migraine. Neurotherapeutics 15:361-370

10. Younis S, Hougaard A, Vestergaard MB, Larsson HBW, Ashina M (2017) Migraine and magnetic resonance spectroscopy : a systematic review. Curr Opin Neurol 30:246-262 
11. Weiller C, May A, Limmroth V, Jüptner M, Kaube H, Schayck RV et al (1995) Brain stem activation in spontaneous human migraine attacks. Nat Med 1: 658-660

12. Afridi SK, Matharu MS, Lee L, Kaube H, Friston KJ, Frackowiak RSJ et al (2005) A PET study exploring the laterality of brainstem activation in migraine using glyceryl trinitrate. Brain 128:932-939

13. Stankewitz A, May A (2011) Increased limbic and brainstem activity during migraine attacks following olfactory stimulation. Neurology 77:476-482

14. Hougaard A, Amin FM, Christensen CE, Younis S, Wolfram F, Cramer SP et al (2017) Increased brainstem perfusion, but no blood- brain barrier disruption, during attacks of migraine with aura. Brain 140:1633-1642

15. Afridi SK, Giffin NJ, Kaube H, Friston KJ, Ward NS, Frackowiak RSJ et al (2005) A positron emission tomographic study in spontaneous migraine. Arch Neurol 62:1270-1275

16. Foerster BR, Pomper MG, Callaghan BC, Petrou M, Edden RAE, Mohamed MA et al (2013) An imbalance between excitatory and inhibitory neurotransmitters in amyotrophic lateral sclerosis revealed by use of 3-T proton magnetic resonance spectroscopy. JAMA Neurol 70:1009-1016

17. Baker EH, Basso G, Barker PB, Smith MA, Bonekamp D, Horská A (2010) Regional apparent metabolite concentrations in young adult brain measured by $1 \mathrm{H}$ MR spectroscopy at 3 tesla. J Magn Reson Imaging 27 489-499

18. Adanyeguh IM, Henry P-G, Nguyen TM, Rinaldi D, Jauffret C, Valabregue R et al (2015) In vivo neurometabolic profiling in patients with spinocerebellar ataxia types 1, 2, 3, and 7. Mov Disord 30:662-670

19. Öz G, Tkáč I (2011) Short-echo, single-shot, full-intensity proton magnetic resonance spectroscopy for neurochemical profiling at $4 \mathrm{~T}$ : validation in the cerebellum and brainstem. Magn Reson Med 65:901-910

20. Zielman R, Teeuwisse WM, Bakels F, Van der Grond J, Webb A, van Buchem MA et al (2014) Biochemical changes in the brain of hemiplegic migraine patients measured with 7 tesla 1H-MRS. Cephalalgia 34:959-967

21. Eftekhari S, Salvatore CA, Calamari A, Kane SA, Tajti J, Edvinsson L (2010) Differential distribution of calcitonin gene-related peptide and its receptor components in the human trigeminal ganglion. Neuroscience 169:683-696

22. Miller S, Liu H, Warfvinge K, Shi L, Dovlatyan M, Xu C et al (2016) Immunohistochemical localization of the calcitonin gene-related peptide binding site in the primate trigeminovascular system using functional antagonist antibodies. Neuroscience 328:165-183

23. Gómez-Vallejo V, Ugarte A, García-Barroso C, Cuadrado-Tejedor M, Szczupak B, Dopeso-Reyes IG et al (2016) Pharmacokinetic investigation of sildenafil using positron emission tomography and determination of its effect on cerebrospinal fluid cGMP levels. J Neurochem 136:403-415

24. Headache Classification Committee of the International Headache Society (IHS) (2013) The international classification of headache disorders, 3rd edition (beta version). Cephalalgia 33:629-808

25. Peng S-L, Dumas JA, Park DC, Liu P, Filbey FM, McAdams CJ et al (2014) Age-related increase of resting metabolic rate in the human brain. Neuroimage 98:176-183

26. Soreni N, Noseworthy MD, Cormier T, Oakden WK, Bells S, Schachar R (2006) Intraindividual variability of striatal $1 \mathrm{H}-\mathrm{MRS}$ brain metabolite measurements at 3 T. Magn Reson Imaging 24:187-194

27. Lopez-Rodriguez F, Medina-Ceja L, Wilson CL, Jhung D, Morales-Villagran A (2007) Changes in extracellular glutamate levels in rat orbitofrontal cortex during sleep and wakefulness. Arch Med Res 38:52-55

28. Christiansen P, Henriksen O, Stubgaard M, Gideon P, Larsson HBW (1993) In vivo quantification of brain metabolites by $1 \mathrm{H}-\mathrm{MRS}$ using water as an internal standard. Magn Reson Imaging 11:107-118

29. Danbolt NC (2001) Glutamate uptake. Prog Neurobiol 65:1-105

30. Hunsberger HC, Konat GW, Reed MN (2017) Peripheral viral challenge elevates extracellular glutamate in the hippocampus leading to seizure hypersusceptibility. J Neurochem 141:341-346

31. Hunsberger HC, Wang D, Petrisko TJ, Alhowail A, Setti SE, Suppiramaniam V et al (2016) Peripherally restricted viral challenge elevates extracellular glutamates and enhances synaptic transmission in the hippocampus. J Neurochem 138:307-316

32. Tkáč I, Öz G, Adriany G, Uǧurbil K, Gruetter R (2009) In vivo 1H NMR spectroscopy of the human brain at high magnetic fields: metabolite quantification at 4T vs. 7T. Magn Reson Med 62:868-879

33. Milman HA, Arnold SB (2002) Neurologic, psychological, and aggressive disturbances with sildenafil. Ann Pharmacother 36:1129-1134
34. Moreira SG, Brannigan RE, Spitz A, Orejuela FJ, Lipshultz LI, Kim ED (2000) Side-effect profile of sildenafil citrate (Viagra) in clinical practice. Urology 4295:474-476

35. Schultheiss D, Müller SV, Nager W, Stief CG, Schlote N, Jonas U et al (2001) Central effects of sildenafil (Viagra) on auditory selective attention and verbal recognition memory in humans: a study with event-related brain potentials. World J Urol 19:46-50

36. Nichols DJ, Muirhead GJ, Harness JA (2002) Pharmacokinetics of sildenafil after single oral doses in healthy male subjects: absolute bioavailability, food effects and dose proportionality. Br J Clin Pharmacol 53:5S-12S

37. Mangia S, Giove F, Tkáč I, Logothetis NK, Henry P-G, Olman CA et al (2009) Metabolic and hemodynamic events after changes in neuronal activity: current hypotheses, theoretical predictions and in vivo NMR experimental findings. J Cereb Blood Flow Metab 29:441-463

38. Lemire J, Mailloux RJ, Appanna VD (2008) Mitochondrial lactate dehydrogenase is involved in oxidative-energy metabolism in human astrocytoma cells (CCF-STTG1). PLoS One 3:1-10

39. Lange T, Dydak U, Roberts TPL, Rowley HA, Bjeljac M, Boesiger P (2006) Pitfalls in lactate measurements at 3T. Am J Neuroradiol 27:895-901

40. Petersen KA, Birk S, Lassen LH, Kruuse C, Jonassen O, Lesko L et al (2005) The CGRP-antagonist, BIBN4096BS does not affect cerebral or systemic haemodynamics in healthy volunteers. Cephalalgia 25:139-147

41. Eftekhari S, Salvatore CA, Johansson S, Chen T, Zeng Z, Edvinsson L (2015) Localization of CGRP, CGRP receptor, PACAP and glutamate in trigeminal ganglion. Relation to the blood-brain barrier. Brain Res 1600:93-109

42. Asghar MS, Hansen AE, Larsson HBW, Olesen J, Ashina M (2012) Effect of CGRP and sumatriptan on the BOLD response in visual cortex. J Headache Pain 13:159-166

43. Bednařík P, Tkáč I, Giove F, DiNuzzo M, Deelchand DK, Emir UE et al (2015) Neurochemical and BOLD responses during neuronal activation measured in the human visual cortex at 7 tesla. J Cereb Blood Flow Metab 35:601-610

44. Schaller B, Xin L, O'Brien K, Magill AW, Gruetter R (2014) Are glutamate and lactate increases ubiquitous to physiological activation? A $1 \mathrm{H}$ functional MR spectroscopy study during motor activation in human brain at 7 tesla. Neuroimage 93:138-145

45. Asghar MS, Becerra L, Larsson HBW, Borsook D, Ashina M (2016) Calcitonin gene-related peptide modulates heat nociception in the human brain - an fMRI study in healthy volunteers. PLoS One 11:1-20

46. Helms G, Piringer A (2001) Restoration of motion-related signal loss and line-shape deterioration of proton MR spectra using the residual water as intrinsic reference. Magn Reson Med 46:395-400

47. Nacewicz BM, Angelos L, Dalton KM, Fischer R, Anderle MJ, Alexander AL et al (2012) Reliable non-invasive measurement of human neurochemistry using proton spectroscopy with an anatomically defined amygdala-specific voxel. Neuroimage 59:2548-2559

48. Allaïli N, Valabrègue R, Auerbach EJ, Guillemot V, Yahia-Cherif L, Bardinet E et al (2015) Single-voxel $1 \mathrm{H}$ spectroscopy in the human hippocampus at $3 \mathrm{~T}$ using the LASER sequence: characterization of neurochemical profile and reproducibility. NMR Biomed 28:1209-1217

49. Cai $K$, Nanga RPR, Lamprou L, Schinstine C, Elliott M, Hariharan $\mathrm{H}$ et al (2012) The impact of gabapentin administration on brain GABA and glutamate concentrations: a 7T 1H-MRS study. Neuropsychopharmacology $37: 2764-2771$

\section{Submit your manuscript to a SpringerOpen ${ }^{\circ}$ journal and benefit from:}

- Convenient online submission

- Rigorous peer review

- Open access: articles freely available online

- High visibility within the field

Retaining the copyright to your article

Submit your next manuscript at $>$ springeropen.com 